\title{
Serum carotenoids and radiographic knee osteoarthritis: the Johnston County Osteoarthritis Project
}

\author{
Anneclaire J De Roos ${ }^{1}$, Lenore Arab ${ }^{2}$, Jordan B Renner ${ }^{3}$, Neal Craft ${ }^{4}$, Gheorghe Luta ${ }^{5}$, \\ Charles G Helmick ${ }^{6}$, Marc C Hochberg ${ }^{7}$ and Joanne M Jordan ${ }^{8, *}$ \\ ${ }^{1}$ National Cancer Institute, Bethesda, MD, USA: ${ }^{2}$ Thurston Arthritis Research Center, Department of Epidemiology \\ and Nutrition, University of North Carolina School of Public Health, Chapel Hill, NC, USA: \\ ${ }^{3}$ Thurston Arthritis Research Center, Department of Radiology, University of North Carolina School of Medicine, \\ Chapel Hill, NC, USA: ${ }^{4}$ Craft Technologies, Inc., Wilson, NC, USA: ${ }^{5}$ Thurston Arthritis Research Center, \\ Department of Biostatistics, University of North Carolina School of Public Health, Chapel Hill, NC, USA: \\ ${ }^{6}$ Centers for Disease Control and Prevention, Atlanta, GA, USA: ${ }^{7}$ Departments of Medicine, Epidemiology, \\ and Preventive Medicine, University of Maryland School of Medicine, Baltimore, MD, USA: ${ }^{8}$ Thurston Arthritis \\ Research Center, 3310 Doc J Thurston Jr Building, CB\# 7330, University of North Carolina, Chapel Hill, \\ NC 27599-7330, USA
}

Submitted 8 September 2000: Accepted 7 February 2001

\begin{abstract}
Objective: Antioxidant intake has been associated with less progression of radiographic knee osteoarthritis (OA), but studies of carotenoid biomarkers and OA have not been done. We examined associations between serum concentrations of nine naturally occurring carotenoids and radiographic knee OA.

Design: The study design was matched case-control. Sera were analysed by highperformance liquid chromatography for nine carotenoids: lutein, zeaxanthin, $\alpha$ - and $\beta$-cryptoxanthin, trans- and cis-lycopene, $\alpha$-carotene, and trans- and cis- $\beta$-carotene. Conditional logistic regression was used to estimate the association between tertiles of each carotenoid and radiographic knee OA, independent of body mass index, education, serum cholesterol, and the other carotenoids.

Setting: Johnston County, North Carolina, United States of America.

Subjects: Two-hundred cases with radiographic knee OA (Kellgren-Lawrence grades $\geq 2$ ) and 200 controls (Kellgren-Lawrence grade $=0$ ) were randomly selected from the Johnston County Osteoarthritis Project, and were matched on age, gender and race.

Results: Participants with serum levels of lutein or $\beta$-cryptoxanthin in the highest tertile were approximately $70 \%$ less likely to have knee OA than controls \{odds ratio (OR) $[95 \%$ confidence interval $(\mathrm{CI})]=0.28[0.11,0.73]$ and $0.36[0.14,0.95]$, respectively\}. Those in the highest tertile of trans- $\beta$-carotene (OR $=6.40[1.86$, 22.1]) and zeaxanthin $(\mathrm{OR}=3.06[1.19,7.85])$ were more likely to have knee OA. Conclusions: While certain carotenoids may protect against knee OA, others may increase the odds of knee OA. Further study of carotenoids and knee OA are warranted before clinical recommendations about these substances and knee OA can be made.
\end{abstract}

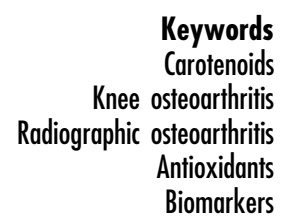

Osteoarthritis (OA) is the most common chronic medical condition in people aged 65 years and older, affecting approximately $85 \%$ of adults aged $75-79$ years ${ }^{1}$. The disease is characterised by loss of cartilage in joints such as knees or hips, and is commonly associated with pain, restricted mobility and functional decline. OA is often regarded as a condition that occurs as part of the natural ageing process. But in fact, the changes in the joint that occur in $\mathrm{OA}$ are quite different from those of normal ageing, and there seem to be many factors contributing to variation in the occurrence and extent of the OA process ${ }^{2}$.

Dietary factors have been shown to modulate the expression and severity of OA in animal models, in which high doses of vitamin $\mathrm{C}$ prevented progression of surgically induced OA in guinea pigs $^{3}$. One postulated mechanism by which certain micronutrients mediate OA incidence and progression is through their antioxidant properties ${ }^{4}$. Reactive oxygen species are generated by 
cells within joints and can cause oxidative damage to various macromolecules. For example, free radicals react with hyaluronic acid, which forms the central axis of proteoglycans in cartilage and maintains the viscosity of synovial fluid within joints. The reaction results in depolymerisation of the macromolecule, leading to destabilisation of connective tissue and loss of synovial fluid viscosity ${ }^{5}$. Similar oxidative reactions with lipids, proteins and DNA may contribute to OA development and progression.

The human body has an extensive line of antioxidant defences in which enzymes and micronutrients quench reactive oxygen species by deoxygenation, thereby rendering these free species harmless ${ }^{6}$. Antioxidant enzymes such as superoxide dismutase are the primary defences in the intracellular space, while micronutrients play a more important role in the intercellular space where antioxidant enzymes are sparse ${ }^{7}$. Micronutrients with known antioxidant properties include $\alpha$-tocopherol and carotenoids, although the antioxidant activity of these compounds in vivo is unproven ${ }^{8,9}$. The relative antioxidant potentials of isomer configurations of these micronutrients, including $\beta$ - and $\alpha$-carotene, or the differences between cis and trans forms of micronutrients remain unclear ${ }^{8,9}$.

The relationship between OA and dietary antioxidants in humans has not received extensive study to date. The Framingham Osteoarthritis Study examined the effect of antioxidant micronutrient intake on the incidence and progression of radiographic knee $\mathrm{OA}^{10}$. High interim dietary intakes of vitamin $\mathrm{C}$ or $\beta$-carotene were found to be associated with $60-70 \%$ decreased risk of cartilage loss over an 8- to 10-year follow-up period. A 30\% reduction in OA progression was also seen for persons who consumed diets rich in vitamin E. Contrary to the findings for $\mathrm{OA}$ progression, $\mathrm{OA}$ incidence did not appear to be affected by reported dietary intakes of $\beta$ carotene, vitamin $\mathrm{C}$ or vitamin $\mathrm{E}$. These data indicate that the OA process might be modified by external factors such as micronutrient intake.

An ongoing study of OA, the Johnston County Osteoarthritis Project, offers another opportunity to study the OA-micronutrient relationship. This project is a community-based longitudinal study of knee and hip OA in a rural North Carolina county. We examined associations between carotenoids and OA by performing a case-control study of serum levels of nine carotenoids and prevalent radiographic knee OA within this population. The serum micronutrient measure circumvents difficulties in measurement of dietary intake as well as inter-individual differences in absorption and metabolism, and may be more useful as a biological marker of internal dose $^{11}$. We measured nine carotenoids in serum, with distinction between different isomer configurations and cis and trans forms.

An association between serum antioxidants and OA might suggest preventive measures for persons who have a suspected predisposition to OA, such as obese persons, those suffering joint injury, or persons with a family history of OA. In addition, intervention in the early stages of OA by dietary modification or micronutrient supplementation might offer a feasible means of preventing progression into severe disease stages. Dietary intervention may be particularly relevant for preventing OA in older persons, who are susceptible to inadequate nutrition $^{12,13}$.

\section{Subjects and methods}

\section{Study population}

The Johnston County Osteoarthritis Project is an ongoing community-based cohort study of knee and hip OA in African-Americans and Caucasians in a rural North Carolina county. The sampling methods and study protocol have been described in detail elsewhere ${ }^{14}$. Briefly, participants were recruited by probability sampling of streets of Johnston County, North Carolina, with deliberate oversampling of African-Americans. All civilian, non-institutionalised, African-American or Caucasian adults aged 45 years and older who were physically and mentally capable of completing the study protocol, including two home interviews and a clinic visit, were eligible. The estimated response rate at the time this nested sample was chosen was 66\%, with no differences according to age, gender, race, or presence of knee pain between respondents and non-respondents ${ }^{14}$. All analyses in this paper used data obtained from the baseline evaluation of study participants.

\section{Etbics}

All procedures in this study were approved by the Institutional Review Boards of the University of North Carolina Schools of Medicine and Public Health.

\section{Sample selection}

Radiographic knee OA was defined from weight-bearing bilateral anteroposterior radiographs of the knee, according to the Kellgren and Lawrence $(\mathrm{K}-\mathrm{L})$ grading scheme: $0=$ no $\mathrm{OA} ; \quad 1=$ questionable $\mathrm{OA} ; 2=$ mild $\mathrm{OA}$; $3=$ moderate $\mathrm{OA} ;$ and $4=$ severe $\mathrm{OA}^{15}$. Cases were randomly selected from those participants having $\mathrm{K}-\mathrm{L}$ grade 2, 3 or 4 in either knee, stratifying on race prior to random selection so that 100 African-American and 100 Caucasian subjects were selected. Controls were randomly selected from those participants with $\mathrm{K}-\mathrm{L}$ grade 0 in both knees, and were matched to cases by age $( \pm 1$ year), gender and race.

\section{Measures}

\section{Questionnaires}

Each subject completed two interviewer-administered questionnaires in his/her home, the first upon recruitment 
and the second during a subsequent home visit. Information of interest for this study included demographics, current alcohol consumption and current smoking status.

\section{Radiographs}

Knee radiographs were read blindly by a single bone and joint radiologist (JBR) for $\mathrm{K}-\mathrm{L}$ grade and for individual radiographic features, using standard atlases ${ }^{15,16}$. Interand intra-rater reliabilities for the radiologist were high, as reported previously ${ }^{14}$.

\section{Clinical procedures}

Height was measured in centimetres and weight in kilograms. Five cubic centimetres of non-fasting blood were collected by venipuncture. Following aspiration, the samples were immediately spun down and $1 \mathrm{~cm}^{3}$ aliquots of serum were drawn off into opaque cryotubes without preservatives, for total serum cholesterol determination by modified Trindar reaction ${ }^{17}$ and subsequent frozen storage at $-86^{\circ} \mathrm{C}$.

\section{Nutrient analyses}

Banked serum samples for the 400 participants were packed in dry ice and shipped to Craft Technologies, Inc. in Wilson, North Carolina for assessment of concentrations of lipid-soluble carotenoids in serum. The panel of carotenoids analysed included lutein, zeaxanthin, $\alpha$ - and $\beta$-cryptoxanthin, trans- and cis-lycopene, $\alpha$-carotene, and trans- and cis- $\beta$-carotene.

The compounds were identified and quantified using high-performance liquid chromatography (HPLC). For serum extraction and HPLC methods, a modification of the procedures described by Nomura et al. ${ }^{18}$ was used. Briefly, after thawing, $150 \mu \mathrm{l}$ aliquots of serum were diluted with $150 \mu \mathrm{l}$ of water and deproteinated by vortexing with $300 \mathrm{ml}$ of ethanol containing tocol as an internal standard and butylated hydroxytoluene as an antioxidant. The samples were extracted twice with $1 \mathrm{ml}$ of hexane; the combined supernatant was evaporated under nitrogen. The residue was then dissolved with vortexing in $35 \mu \mathrm{l}$ of ethyl acetate, diluted with $100 \mu \mathrm{l}$ of mobile phase, and agitated ultrasonically for $15 \mathrm{~s}$ prior to placement in the autosampler. A $15 \mu \mathrm{l}$ volume was injected.

The HPLC system consisted of a computer data system, a solvent degasser, an autosampler maintaining samples at $20^{\circ} \mathrm{C}$, a Spherisorb ODS2 column $(3 \mu \mathrm{m}$, $4.6 \mathrm{~mm} \times 150 \mathrm{~mm}$ with titanium frits), a guard column containing the same stationary phase, a column heater at $29^{\circ} \mathrm{C}$, a programmable ultraviolet/visible detector to measure retinol at $325 \mathrm{~nm}$ and carotenoids at $450 \mathrm{~nm}$, and a programmable fluorescence detector to measure tocol and tocopherols at $296 \mathrm{~nm}$ excitation/340 nm emission. The separation was performed isocratically using a mobile phase of $83 \%$ acetonitrile/13\% dioxane/
4\% methanol containing $150 \mathrm{mM}$ ammonium acetate and $0.1 \%$ triethylamine at a flow rate of $1.5 \mathrm{ml} \mathrm{min}^{-1}$.

\section{Quality control}

In-house quality control (QC) samples were analysed at the beginning, end, and at 24 sample intervals. Linear calibration curves were prepared consisting of multiple concentrations of analytes which spanned the physiological levels of the analysed carotenoids in serum. The calibrants included lutein, zeaxanthin, $\alpha$ - and $\beta$-cryptoxanthin, lycopene, $\alpha$ - and $\beta$-carotene. Serum quantification was performed by internal standard calibration using peak area ratios. The relative standard deviation of analytes in QC samples ranged from 3 to $10 \%$.

Ten per cent of all samples $(n=40)$ were submitted to the laboratory as duplicate 'phantom samples', labelled with a new identification number. The repeatability of the laboratory protocol was evaluated for each carotenoid by examination of scatter plots of assay results for the 40 phantom samples plotted against their 40 duplicate samples, and calculation of Pearson correlation coefficients. Pearson correlation coefficients ranged between 0.83 and 0.99 . Except for $\alpha$-cryptoxanthin $(r=0.83)$, the correlation coefficients were all greater than 0.90 , and only these were included in statistical analyses.

\section{Statistical analyses}

Analyses were done on a microcomputer using SAS, version 6.12 (Cary, NC). Mean serum carotenoid levels were compared across case and control groups by calculating a $t$-statistic with its $P$-value, after log-transformation of the skewed data produced a more symmetrical distribution. Crude and adjusted odds ratios (ORs) and 95\% confidence intervals (95\% CIs) for each OA-carotenoid association were then estimated using unadjusted and multivariable conditional logistic regression models, respectively, comparing cases and controls across carotenoid tertiles, using the lowest tertile as a referent category. Potential confounders of a priori interest included education ( $<$ high school graduate/ $\geq$ high school graduate), current smoking (yes/no), current alcohol consumption (yes/no), body mass index (BMI in $\mathrm{kg} \mathrm{m}^{-2}$ ) and total serum cholesterol $\left(\mathrm{mg} \mathrm{dl}^{-1}\right)$. These factors have been shown to be associated with levels of lipid-soluble nutrients in serum $^{19}$, and are either known or suspected to be independent factors associated with knee $\mathrm{OA}^{20}$. Each covariate was assessed for its importance as a confounder of each OA-carotenoid relationship by the percentage change in the odds ratios for the carotenoid effect when that covariate was removed from the model. Changes in risk estimates of greater than 5\% between nested models were considered to indicate confounding of the carotenoid effect by that covariate.

Because a number of the carotenoids studied often occur together in foods, the serum levels of one are likely 
Table 1 Selected characteristics of subjects

\begin{tabular}{|c|c|c|c|}
\hline & Cases $^{*}(n=200)$ & Controls $(n=200)$ & \\
\hline Characteristic & Mean (SD) or \% & Mean (SD) or \% & OR $(95 \% \mathrm{Cl})$ \\
\hline Education less than high school graduate & $52.0 \%$ & $39.0 \%$ & $1.80(1.15-2.81)$ \\
\hline Currently smokes & $14.5 \%$ & $20.5 \%$ & $0.72(0.39-1.32)$ \\
\hline Currently drinks alcohol & $17.0 \%$ & $21.0 \%$ & $0.70(0.40-1.22)$ \\
\hline Body mass index (BMI) $\left(\mathrm{kg} \mathrm{m}^{-2}\right)$ & $32.00(7.19)$ & $27.05(4.99)$ & $1.17(1.11-1.22)$ \\
\hline Total serum cholesterol $\left(\mathrm{mmol}^{-1}\right)$ & $5.72(1.21)$ & $5.61(1.14)$ & $1.00(0.99-1.01)$ \\
\hline
\end{tabular}

* $68.5 \%$ (137) of cases had Kellgren-Lawrence radiographic grade 2; 23\% (46) had Kellgren-Lawrence grade 3; and 8.5\% (17) had Kellgren-Lawrence grade 4.

to be associated with those of another. For this reason, confounding of each OA-carotenoid association by other carotenoids is possible. We therefore further examined each OA-carotenoid association in one multivariable model which controlled for the effects of the covariates and all other carotenoids simultaneously. All carotenoids were entered into the model as tertile categories, using the lower tertile as a referent group. Confounding by covariates was reassessed for this model, using the same criteria previously described. Potential collinearity of the micronutrient variables was assessed with regression diagnostics, similar to those used for linear regression ${ }^{21}$ Models were considered acceptable with no serious collinearity between predictors if the following criteria were met: variance inflation factor values did not exceed 10.0, and condition index values in a principal components analysis of the predictors were each less than 30.0 None of the models violated these criteria.

In order to assess a linear trend, we analysed each carotenoid as a continuous variable consisting of the original measured serum concentrations in a multivariable conditional logistic regression model including the covariates and all other carotenoid tertiles. Odds ratios and $95 \%$ confidence intervals were calculated to reflect the OA-carotenoid association corresponding to an increase in serum level as seen from the 10th to the 90th carotenoid percentile. $P$-values less than 0.20 were considered to indicate a linear trend.

\section{Results}

The distribution of selected characteristics in the study population is shown in Table 1. Matching ensured equal distributions of age, gender and race between the cases and controls. In total, 200 African-Americans and 200 Caucasians were selected, with two-thirds female. Ages of subjects ranged from 45 to 92 years, with a mean [standard deviation (SD)] of 62.4 (10.3) years. Compared with controls, cases were less likely to have completed high school $(P=0.01)$ and had a higher mean BMI $(P=0.0001)$, but were otherwise similar.

Geometric means of serum carotenoid concentrations by case or control status are presented in Table 2, listed in decreasing order. Mean serum levels of trans- and cislycopene, lutein, $\alpha$-carotene and $\beta$-cryptoxanthin were slightly lower among cases than controls.

The associations between serum carotenoid tertiles and radiographic knee OA are presented in Table 3. Unadjusted analyses of individual carotenoids revealed inverse associations between radiographic knee OA and cislycopene, lutein and $\beta$-cryptoxanthin, in which subjects in the highest tertiles of these carotenoids were approximately 35-45\% less likely to have radiographic knee OA. After adjustment for body mass index, education and total serum cholesterol, the inverse associations remained but were diluted (data not shown). Current smoking and alcohol consumption did not confound the association

Table 2 Geometric means of serum carotenoid concentrations $\left(\mu \mathrm{mol} \mathrm{I}^{-1}\right)$ by radiographic knee osteoarthritis

\begin{tabular}{lcccc}
\hline & \multicolumn{2}{c}{ Cases } & & Controls \\
\cline { 2 - 2 } Carotenoid & $\mathrm{GM}^{*}(95 \% \mathrm{Cl})$ & & $\mathrm{GM}(95 \% \mathrm{Cl})$ & $P$-value† \\
\hline Trans- $\beta$-carotene & $0.257(0.227,0.294)$ & & $0.272(0.242,0.304)$ & 0.56 \\
Trans-lycopene & $0.216(0.194,0.238)$ & & $0.233(0.212,0.257)$ & 0.26 \\
Cis-lycopene & $0.201(0.183,0.220)$ & & $0.216(0.197,0.238)$ & 0.24 \\
Lutein & $0.176(0.162,0.190)$ & & $0.195(0.183,0.209)$ & 0.04 \\
$\beta$-Cryptoxanthin & $0.096(0.087,0.105)$ & & $0.112(0.101,0.121)$ & 0.02 \\
Zeaxanthin & $0.054(0.051,0.058)$ & & $0.056(0.053,0.062)$ & 0.54 \\
$\alpha$-Carotene & $0.045(0.041,0.048)$ & & $0.050(0.045,0.056)$ & 0.13 \\
Cis- $\beta$-carotene & $0.045(0.041,0.048)$ & & $0.041(0.037,0.045)$ & 0.96 \\
\hline
\end{tabular}

* $\mathrm{GM}=$ geometric mean.

† Matched $t$-test based on log-transformed values. 
Table 3 Associations between serum carotenoids and radiographic knee osteoarthritis

\begin{tabular}{|c|c|c|c|c|c|}
\hline Carotenoids & $\begin{array}{l}\text { Tertile lower } \\
\text { cutpoints } \\
\left(\mu \mathrm{mol} \mathrm{I}^{-1}\right)\end{array}$ & $\begin{array}{l}\text { Unadjusted OR } \\
\qquad(95 \% \mathrm{Cl})\end{array}$ & $\begin{array}{l}\text { Adjusted OR } \\
(95 \% \mathrm{Cl})^{*}\end{array}$ & $\begin{array}{l}\text { Adjusted OR }(95 \% \mathrm{Cl}) \\
\text { comparing the } 90 \text { th to } \\
\text { the } 10 \text { th percentile }\end{array}$ & $\begin{array}{l}P \text {-value for } \\
\text { linear trend }\end{array}$ \\
\hline \multicolumn{6}{|l|}{ Trans- $\beta$-carotene } \\
\hline Lower tertile & & 1.00 & 1.00 & $1.78(0.77-4.15)$ & 0.18 \\
\hline Middle tertile & 0.181 & $0.84(0.51-1.39)$ & $1.83(0.81-4.14)$ & & \\
\hline Upper tertile & 0.376 & $0.98(0.57-1.69)$ & $6.40(1.86-22.1)$ & & \\
\hline \multicolumn{6}{|l|}{ Trans-lycopene } \\
\hline Lower tertile & & 1.00 & 1.00 & $1.29(0.36-4.62)$ & 0.69 \\
\hline Middle tertile & 0.183 & $1.13(0.68-1.89)$ & $1.84(0.53-6.36)$ & & \\
\hline Upper tertile & 0.320 & $0.68(0.40-1.17)$ & $1.28(0.30-5.51)$ & & \\
\hline \multicolumn{6}{|l|}{ Cis-lycopene } \\
\hline Lower tertile & & 1.00 & 1.00 & $0.57(0.17-1.90)$ & 0.36 \\
\hline Middle tertile & 0.170 & $0.74(0.45-1.21)$ & $0.54(0.16-1.84)$ & & \\
\hline Upper tertile & 0.289 & $0.62(0.36-1.07)$ & $0.61(0.12-2.95)$ & & \\
\hline \multicolumn{6}{|l|}{ Lutein } \\
\hline Lower tertile & & 1.00 & 1.00 & $0.48(0.24-0.97)$ & 0.04 \\
\hline Middle tertile & 0.151 & $0.66(0.42-1.05)$ & $0.60(0.28-1.27)$ & & \\
\hline Upper tertile & 0.229 & $0.55(0.33-0.92)$ & $0.28(0.11-0.73)$ & & \\
\hline \multicolumn{6}{|l|}{$\beta$-Cryptoxanthin } \\
\hline Lower tertile & & 1.00 & 1.00 & $0.59(0.30-1.13)$ & 0.11 \\
\hline Middle tertile & 0.074 & $0.65(0.40-1.06)$ & $0.60(0.28-1.28)$ & & \\
\hline Upper tertile & 0.128 & $0.55(0.64-0.91)$ & $0.36(0.14-0.95)$ & & \\
\hline \multicolumn{6}{|l|}{ Zeaxanthin } \\
\hline Lower tertile & & 1.00 & 1.00 & $1.22(0.54-2.74)$ & 0.63 \\
\hline Middle tertile & 0.047 & $0.98(0.62-1.57)$ & $2.28(1.06-4.94)$ & & \\
\hline Upper tertile & 0.070 & $1.02(0.63-1.63)$ & $3.06(1.19-7.85)$ & & \\
\hline \multicolumn{6}{|l|}{$\alpha$-Carotene } \\
\hline Lower tertile & & 1.00 & 1.00 & $1.04(0.70-1.53)$ & 0.86 \\
\hline Middle tertile & 0.032 & $1.04(0.64-1.71)$ & $1.06(0.50-2.23)$ & & \\
\hline Upper tertile & 0.058 & $0.87(0.52-1.43)$ & $1.47(0.63-3.46)$ & & \\
\hline \multicolumn{6}{|l|}{ Cis- $\beta$-carotene } \\
\hline Lower tertile & & 1.00 & 1.00 & $0.70(0.25-1.92)$ & 0.48 \\
\hline Middle tertile & 0.028 & $0.96(0.59-1.55)$ & $1.06(0.48-2.36)$ & & \\
\hline Upper tertile & 0.052 & $0.95(0.56-1.62)$ & $0.66(0.22-1.97)$ & & \\
\hline
\end{tabular}

* Adjusted models included BMI, education, total serum cholesterol, all carotenoids.

with OA for any of the carotenoids and were removed from the models.

In the multivariable model simultaneously including covariates and all carotenoid tertiles, inverse associations between knee OA and lutein and $\beta$-cryptoxanthin were strengthened, such that those with serum levels in the upper tertiles of these carotenoids were approximately $70 \%$ less likely to have radiographic knee OA. In addition, those in the highest tertile of trans- $\beta$-carotene and zeaxanthin were more likely to have knee OA.

In the models with individual carotenoids as continuous variables, the inverse associations of lutein and $\beta$ cryptoxanthin with knee OA appeared to follow a linear trend, while a positive linear trend was indicated for trans- $\beta$-carotene, consistent with the results noted with these carotenoids defined as tertiles.

\section{Discussion}

This is the first study to evaluate serum levels of carotenoids and knee OA. Except for $\beta$-carotene, none of the compounds examined in this study has been previously evaluated for involvement in the OA disease process. We hypothesised that serum concentrations of antioxidant carotenoids would be inversely associated with radiographic knee OA, because of the potential for these compounds to quench oxygen free radical species which may be involved in joint damage. Our data indicated that serum levels of lutein and $\beta$-cryptoxanthin were inversely associated with radiographic knee OA, while cases were more likely than controls to have higher serum levels of trans- $\beta$-carotene and zeaxanthin. No consistent association between knee OA and the other carotenoids was seen.

Lutein has been the subject of considerable research as an antioxidant, particularly in the context of age-related macular degeneration, in which a low density of macular pigment is thought to be a risk factor ${ }^{22}$. Lutein has been shown to increase the level of macular pigment and has also been shown to protect liver cells from oxidative damage and inhibit mammary tumour growth in mice ${ }^{22-28}$. The cryptoxanthins, including $\beta$-cryptoxanthin, have also been the focus of research in light of their potential antioxidant properties ${ }^{26-28}$. However, the effects of lutein or $\beta$-cryptoxanthin upon cartilage or other joint components have not been evaluated.

Although our findings of positive associations between knee $\mathrm{OA}$ and $\beta$-carotene and zeaxanthin differ from our 
hypothesis, these associations are not biologically implausible, given the current lack of extensive knowledge concerning the environment within the joint. It is known that carotenoids can have both antioxidant and prooxidant properties, depending on the oxygen concentration of the subcellular environment ${ }^{8}$. Depending on the oxygen pressure and bioavailability of compounds within the joint, a carotenoid such as $\beta$-carotene could potentially promote rather than prevent lipid peroxidation. The fact that those receiving $\beta$-carotene supplementation in clinical trials had an increased incidence of lung cancer $^{29,30}$ illustrates the complexity of biological activity of this carotenoid in vivo.

Our findings in reference to $\beta$-carotene differ from those of the Framingham cohort, in which low intake of $\beta$-carotene was associated with knee OA progression, but not incidence ${ }^{10}$. Differences between the two studies are likely the result of differing study designs, exposure measures and adjustment for confounders. First, the $\beta$ carotene-knee OA association was examined longitudinally within the Framingham cohort, utilising interim intake of this antioxidant as assessed by food-frequency questionnaire. Our cross-sectional study, lacking detailed dietary data, examined OA prevalence and used serum $\beta$-carotene as an exposure measure. Although there are clear advantages to each nutritional exposure measure, the use of serum levels avoids dietary recall error and varying amounts of carotenoids in foods between regions, seasons and cooking practices 9 . The serum measure also accounts for differences in inter-individual absorption and metabolism and may be more reflective of a biologically active internal dose ${ }^{11}$. In addition, our study did not control for potential confounding by vitamin $\mathrm{C}$, which was inversely associated with knee OA progression in the Framingham study ${ }^{10}$.

There are several possible explanations why other hypothesised inverse associations with knee OA in our study may have been obscured if they do in fact exist. First, the cross-sectional nature of the data presents difficulty in determining whether observed associations are indicative of disease prevention or are rather a consequence of disease. Complex relationships exist between relevant variables, in that health factors and behaviours potentially leading to OA (nutrient intake, BMI) may also change as a result of the onset of pain and functional loss associated with OA. Data from the southeastern United States show that persons who take medications for OA are more likely to take vitamin supplements than those who do not report taking such medications ${ }^{31}$. Such behavioural changes in dietary or supplemental nutrient intake, had they occurred in our study participants, could potentially explain the positive associations observed for trans- $\beta$-carotene, in which subjects with serum concentrations in the upper tertile were approximately six times as likely to have knee OA. Although we did not have detailed data on dietary or supplemental nutrient intake in our study, our analyses done among subjects reporting knee pain did not support such an explanation. Despite the limited inference possible from the cross-sectional study design, it should be noted that similar use of cross-sectional measures of serum micronutrients has proved informative in studies of cardiovascular disease, in which prospective studies have later corroborated cross-sectional results ${ }^{32,33}$.

Second, the measure of carotenoids in serum may be reflective of, but not necessarily specific to, carotenoid levels at the site of action. There is evidence that cells within joints, such as chondrocytes in cartilage, are active producers of reactive oxygen species, suggesting that combative antioxidant activity may be important within the joints themselves. One study indicated that $\alpha$ tocopherol levels in the synovial fluid of patients with inflammatory joint disease were significantly lower than the level in matched serum of the same patient ${ }^{34}$, suggesting that the consumption of $\alpha$-tocopherol may be localised at the site of oxidative activity. Although we cannot determine whether our serum measures reflect the carotenoid concentrations in the joint, we can thus conjecture that larger differences in levels might have existed within knee joints than in serum.

Third, as above, potentially uncontrolled confounding in our study, in particular by vitamin $\mathrm{C}$, could influence the associations we noted (or failed to observe) between carotenoids and knee OA. Fruit and vegetables containing carotenoids usually also contain high concentrations of vitamin $\mathrm{C}$, resulting in a strong correlation between these compounds in serum. Vitamin $\mathrm{C}$ is a potent antioxidant in vivo, and may additionally protect against OA by other mechanisms such as stimulating increased collagen and proteoglycan synthesis ${ }^{7,35}$. Because we did not have data on serum vitamin $C$ levels in our study, the effect of this micronutrient on knee $\mathrm{OA}$, and on the relationships between carotenoids and knee OA, could not be assessed.

In addition, our cases were defined on the basis of having at least one knee with Kellgren-Lawrence radiographic grade 2 . This defines $\mathrm{OA}$ by the presence of a definite osteophyte but does not require joint space narrowing. Unfortunately, we did not have a sufficient number of informative pairs with Kellgren-Lawrence grades 3 or 4 in this matched sample to allow separate analysis according to a stricter $\mathrm{OA}$ definition requiring definite joint space narrowing, or to test whether there was a significant difference in the relationships with carotenoids between grades 2 and grades 3 and 4 . Similarly, analysis according to the individual radiographic features of osteophytes and joint space narrowing was precluded, because insufficient numbers in the more severe categories made meaningful comparisons impossible.

Despite its limitations, our study had several significant strengths, particularly in its ethnically diverse, 
community-based sample and biological exposure measure. Our data provide some evidence that circulating levels of certain carotenoids are inversely associated with prevalent radiographic knee OA, implying a potentially protective effect for these compounds. However, the complex relationships between serum carotenoid levels and the OA disease process remain to be elucidated and should be examined prospectively. Future studies should also consider the most representative measure of antioxidant activity specific to the site of action. Given the significant impact of $\mathrm{OA}$ and the promising but small body of knowledge about its relationship to nutritional factors, the effect of antioxidants on the incidence and progression of OA is worthy of further study.

\section{Acknowledgements}

The authors wish to thank Janice Woodard, Wilma Howard, Kyle McDermott, and Drs Edwin Hartman and Kim Shaftner for their help in implementing the Johnston County Osteoarthritis Project protocol in the field, and Dr John Fryer for his help with sampling and encouragement. Supported by Centers for Disease Control and Prevention/Association of Schools of Public Health grant SO43; National Institute of Arthritis, Musculoskeletal, and Skin Diseases Multipurpose Arthritis Musculoskeletal Disease Center grant 5-P60AR30701; University of North Carolina Clinical Nutrition Research Center grant DK56350, sponsored by National Institutes of Health.

\section{References}

1 Lawrence RC, Helmick CG, Arnett FC, Deyo RA, Felson DT, Giannini EH, Heyse SP, Hirsch R, Hochberg MC, Hunder GG, Liang MH, Pillemer SR, Steen VD, Wolfe F. Estimates of the prevalence of arthritis and selected musculoskeletal disorders in the United States. Arthritis Rheum. 1998; 41: 778-99.

2 Felson DT, Zhang Y. An update on the epidemiology of knee and hip osteoarthritis with a view to prevention. Arthritis Rheum. 1998; 41: 1343-55.

3 Schwartz ER, Leveille C, Oh WH. Experimentally-induced osteoarthritis in guinea pigs: effect of surgical procedure and dietary intake of vitamin C. Lab. Animal Sci. 1981; 31 : $683-7$.

4 Henrotin Y, Deby-Dupont G, Deby C, Franchimont P, Emerit I. Active oxygen species, articular inflammation and cartilage damage. In: Emerit I, Chance B, eds. Free Radicals and Aging. Switzerland: Birkhauser Verlag Basel, 1992; 308-22.

5 Winrow VR, Winyard PG, Morris CJ, Blake DR. Free radicals in inflammation: second messengers and mediators of tissue destruction. Br. Med. Bull. 1993; 49: 506-22.

6 Cheeseman KH, Slater TF. An introduction to free radical biochemistry. Br. Med. Bull. 1993; 49: 481-93.

7 Frei B. Reactive oxygen species and antioxidant vitamins: mechanisms of action. Am. J. Med. 1994; 97: 5S-13S.

8 Omaye ST, Krinsky NI, Kagan VE, Mayne ST, Liebler DC, Bidlack WR. Beta-carotene: friend or foe? Fund. Appl. Toxicol. 1997; 40: 163-74.
9 Rock CL. Carotenoids: biology and treatment. Pharmacol. Therapeut. 1997; 75: 185-97.

10 McAlindon TE, Jacques P, Zhang Y, Hannan MT, Aliabadi P, Weissman B, Rush D, Levy D, Felson DT. Do antioxidant micronutrients protect against the development and progression of knee osteoarthritis? Arthritis Rheum. 1996; 39: 648-56.

11 Kardinaal AFM, van't Veer P, Brants HAM, van den Berg H, van Schoonhoven J, Hermus RJJ. Relations between antioxidant vitamins in adipose tissue, plasma, and diet. Am. J. Epidemiol. 1995; 141: 440-50.

12 Learner RM, Kivett VR. Discriminators of perceived dietary adequacy among the rural elderly. J. Am. Diet. Assoc. 1981; 78: $330-7$.

13 Kolasa KM, Mitchell JP, Jobe AC. Food behaviors of southern rural community-living elderly. Arch. Fam. Med. 1995; 4: 844-8.

14 Jordan JM, Linder GF, Renner JB, Fryer JG. The impact of arthritis in rural populations. Arthritis Care Res. 1995; 8: 242-50.

15 Kellgren JK, Lawrence JS. The Epidemiology of Chronic Rheumatism: Atlas of Standard Radiographs. Oxford: Blackwell Scientific, 1963.

16 Scott WWJ, Lethbridge-Cejku M, Reichle R, Wigley FM, Tobin JD, Hochberg MC. Reliability of grading scales for individual radiographic features of osteoarthritis of the knee: the Baltimore Longitudinal Study of Aging atlas of knee osteoarthritis. Invest. Radiol. 1993; 28: 497-501.

17 Tietz N. Fundamentals of Clinical Chemistry. Philadelphia, PA: WB Saunders Company, 1976.

18 Nomura A, Stemmermann GN, Lee J, Craft NE. Serum micronutrients and prostate cancer in Japanese Americans in Hawaii. Cancer Epidemiol. Biomark. Prev. 1997; 6: 487-91.

19 Brady WE, Mares-Perlman JA, Bowen P, StacewiczSapuntzakis M. Human serum carotenoid concentrations are related to physiologic and lifestyle factors. J. Nutr. 1996; 126: 129-37.

20 Felson DT, Zhang Y, Hannan MT, Naimark A, Weissman B, Aliabadi P, Levy D. Risk factors for incident radiographic knee osteoarthritis in the elderly: the Framingham Study. Arthritis Rheum. 1997; 40: 728-33.

21 Kleinbaum DG, Kupper LL, Muller KE, Nizam A. Applied Regression Analysis and Multivariable Models. Pacific Grove, CA: Brooks/Cole Publishing Company, 1998.

22 Landrum JT, Bone RA, Joa H, Kilburn MD, Moore LL, Sprague KE. A one year study of the macular pigment: the effect of 140 days of a lutein supplement. Exp. Eye Res. 1997; 65: 57-62.

23 Park JS, Chew BP, Wong TS. Dietary lutein from marigold extract inhibits mammary tumor development in BALB/C mice. J Nutr. 1998; 128: 1650-6.

24 Martin KR, Failla ML, Smith JCJ. Beta-carotene and lutein protect HepG2 human liver cells against oxidant-induced damage. J. Nutr. 1996; 126: 2098-106.

25 Anonymous. The effect of a dietary lack of xanthophyll on the eye of the monkey. Nutr. Rev. 1980; 38: 384-6.

26 Lyle BJ, Mares-Perlman JA, Klein BEK, Klein R, Palta M, Bower PE, Greger JL. Serum carotenoids and tocopherols and incidence of age-related nuclear cataract. Am. J Clin. Nutr. 1999; 69: 272-7.

27 Lyle BJ, Mares-Perlman JA, Klein BEK, Klein R, Greger JL. Antioxidant intake and risk of incident age-related nuclear cataracts in the Beaver Dam Eye Study. Am. J. Epidemiol. 1999; 149: 801-9

28 Howard AN, Williams NR, Palmer CR, Cambon JP, Evans AE, Foote JW, Marques-Vidal P, Mc Crum EE, Ruidavets JB, Nigdikar SV, Rajput-Williams J, Thurnham DI. Do hydroxycarotenoids prevent coronary heart disease? A comparison 
between Belfast and Toulouse. Int. J. Vitamin Nutr. Res. 1996; 66: 113-8.

29 Omenn GS, Goodman GE, Thornquist MD, Balmes J, Cullen MR, Glass A, Keogh JP, Meyskens FL, Valanis B, Williams JH, Barnhart S, Cherniak MG, Brodlin CA, Hammar S. Risk factors for lung cancer and for intervention effects in CARET, the beta-carotene and retinol efficacy trial. I. Natl. Cancer Inst. 1996; 88: 1550-8.

30 The Alpha-Tocopherol, Beta-Carotene Cancer Prevention Study Group. The effect of vitamin E and beta carotene on the incidence on lung cancer and other cancers in male smokers. New Engl. J. Med. 1994; 330: 1029-35.

31 Houston DK, Johnson MA, Daniel TD, Poon LW. Health and dietary characteristics of supplement users in an elderly population. Int. J. Vitamin Nutr. Res. 1997; 67: 183-91.
32 Ness AR, Powles JW, Khaw KT. Vitamin C and cardiovascular disease: a systematic review. J. Cardiovascular Risk 1996; 3: 513-21.

33 Kohlmeier L, Hastings SB. Epidemiologic evidence of a role of carotenoids in cardiovascular disease prevention. Am.J. Clin. Nutr. 1995; 62: 1370S-6S.

34 Fairburn K, Grootveld M, Ward RJ, Abiuka C, Kus M, Williams RB, Winyard PG, Blake DR. Alpha-tocopherol, lipids, and lipoproteins in knee-joint synovial fluid and serum from patients with inflammatory joint disease. Clin. Sci. 1992; 83: 657-64

35 Schwartz ER, Adamy L. Effect of ascorbic acid on arylsulfatase activities and sulfated proteoglycan metabolism in chondrocyte cultures. J. Clin. Invest. 1977; 60: 96106. 